\title{
Accumulation Pattern of Flavonoids in Cabernet Sauvignon Grapes Grown in a Low-Latitude and High-Altitude Region
}

\author{
Ran-Ran Xing, Fei He, Hui-Lin Xiao, Chang-Qing Duan, Qiu-Hong Pan* \\ Centre for Viticulture \& Enology, College of Food Science and Nutritional Engineering, China Agricultural University, Beijing, \\ 100083 China
}

Submitted for publication: May 2014

Accepted for publication: December 2014

Key words: High-altitude region, anthocyanins, flavonols, flavan-3-ols, grape berry

Particular climate conditions in a low-latitude and high-altitude region endow grape berries with distinctive quality characteristics. So far, few reports have been concerned with the formation of berry flavour in such a region. This study aimed to investigate the accumulation pattern of flavonoids in Vitis vinifera $\mathrm{L}$. cv. Cabernet Sauvignon grape berries growing at different altitudes of the highland in southwest China in two consecutive vintages. In addition to the 3-O-monoglucosides and 3-O-acyl monoglucosides of the five main anthocyanidins (delphinidin, cyanidin, peonidin, petunidin and malvidin), some uncommon anthocyanins, such as three diglucosides of anthocyanidins and pelargonidin-3-O-glucoside, were detected in the grape berries. Higher altitude cultivation greatly promoted the production of anthocyanins and flavonols, particularly cyanidintype anthocyanins and quercetin-type flavonols from the F3' $H$ branch of the flavonoid biosynthetic pathway. Flavan-3-ols from both branches were comparatively less influenced by vineyard altitude. Vintage in this high-altitude region also had a dramatic influence on the accumulation of flavonoids. Most of the anthocyanin and flavonol components were affected more by vineyard altitude than by vintage, whereas the accumulation of flavan-3-ols differed mainly between vintages. The present data will not only improve the understanding of flavonoid accumulation in grapes from a high-altitude region with different climates, but also provide practical guidance for the production of high-quality grapes and wine.

\section{INTRODUCTION}

China's wine-producing regions are situated mostly between northern latitudes $35^{\circ}$ and $40^{\circ}$, where the wine output accounts for over $95 \%$ of total national production. These regions all belong to the typical continental monsoon climate featuring cold, dry winters and hot, damp summers, which differ completely from the climate of cold, damp winters and hot, dry summers in other wine-producing regions of the world such as Europe. Only a few wines are produced from a high-attitude area between northern latitudes $28^{\circ}$ and $30^{\circ}$ in southwest China, the most typical of which is the Yunnan plateau region, where the vineyards are located at elevations from $1500 \mathrm{~m}$ to $2900 \mathrm{~m}$ above sea level (asl). The soil there is reddish-brown and rich in minerals. This highland has adequate sunshine, an average rainfall of about $350 \mathrm{~mm}$ per year, a timely dry season from October/November until June and high ultraviolet intensity. The temperatures associated with low latitudes are usually moderated by the high altitude, and vineyards are protected from the ill-effects of the heat by significantly cooler nights. The diurnal temperature variation during the growing season helps to extend the ripening period, allowing grapes to develop flavour along with acidity. Over the past few decades, many Vitis vinifera cultivars, such as Cabernet Sauvignon and Merlot, have been cultivated successfully in this region and have been producing high-quality wines. Now, this wine-producing region of low latitude and high attitude has attracted many wine producers and promises an increasing amount of viticulture.

Flavonoids are a large group of phenolic secondary metabolites that are concentrated in the seeds, skins and stems of grapes (Obreque-Slier et al., 2010). They not only play various functional roles, such as protection against ultraviolet (UV) radiation and the prevention of pigmentation and pathogens (Gómez-Míguez et al., 2006), but also contribute to the colour, stability, astringency and bitterness of wines (Gawel, 1998). Flavonoids also have aroused considerable interest because of their antioxidant properties and potential benefit to human health (SantosBuelga \& Scalbert, 2000). Flavonoids are composed mainly of three subgroups: anthocyanins, flavonols and flavan-3-ols, of which anthocyanins and flavonols are critical components contributing to the quality of red grapes and wines. These two groups of compounds exist predominantly in the skins of red grapes, whereas flavan-3-ols are present in both the seeds and skins of the grape berry (Koyama et al., 2012; Zarrouk et al., 2012). Anthocyanins in Vitis vinifera cultivars consist of five aglycones: cyanidin, delphinidin, peonidin, petunidin and malvidin, almost all of which are in the form of

*Corresponding author: E-mail address: panqh@cau.edu.cn [Tel: 86-10-62736191; Fax: 86-10-62738658]

Acknowledgements: The authors want to thank Yunan Shangeli-La Winery, Yunnan Province, China, for their kind help with grape sampling. Thanks also go to the Yunan Shangeli-La Winery and the National Natural Science Foundation of China (Grant No. 30971980), for their funding support 
3 -glycosides. Anthocyanins are responsible for the red, purple and blue colours of grape berries (Picariello et al., 2012). The variation in anthocyanin composition will significantly affect the colour presentation of the grape berry and wine. Flavonols in grapes exist mostly in the form as 3-glycosides of kaempferol, quercetin, myricetin and isorhamnetin (3'-methylation of quercetin), whereas their corresponding free aglycones can be found in the wine (Castillo-Muñoz et al., 2007). Flavonols are a group of yellow pigments and act as prominent cofactors involved in colour enhancement by co-pigmentation (Castillo-Muñoz et al., 2009). Flavan3 -ols mainly comprise (-)-epicatechin-3-O-gallate in the seeds and $(+)$-catechin, (-)-epigallocatechin-3-O-gallate, (-)-epicatechin and (-)-epicatechin-3-O-gallate in the skins of the grape berry, and these constitute the basic building blocks of grape tannins and endow wine with astringency, bitterness and texture (Dixon et al., 2005). Accordingly, the composition and concentration of flavonoids in the grape berry to a large extent determine the sensorial quality of the wine produced.

Flavonoids are synthesised by two parallel pathways in the grape berry (Davies \& Schwinn, 2003; Winkel-Shirley, 2001). The one is a flavonoid $3^{\prime}$-hydroxylase (F3' $\left.\mathrm{H}\right)$-mediated branch pathway by which the $3^{\prime}$ position of the B-ring of flavonoids is hydroxylated to produce $3^{\prime}, 4^{\prime}$-hydroxylated flavonoids (also named 3',4'-substituted compounds); and the other is a flavonoid $3^{\prime}, 5^{\prime}$-hydroxylase $\left(\mathrm{F}^{\prime} 5^{\prime} \mathrm{H}\right)$-mediated branch that produces $3^{\prime}, 4^{\prime}, 5^{\prime}$-hydroxylated flavonoids (also named 3',4',5'-substituted compounds) (Obreque-Slier et al., 2010). 3',4'-substituted flavonoids are composed mainly of quercetin-type flavonols, cyanidin-type anthocyanins, as well as catechin (C), epicatechin (EC) and (-)-epicatechin-3-Ogallate (ECG), whereas 3',4',5'-substituted flavonoids include myricetin-type flavonols, delphinidin-type anthocyanins and epigallocatechin (EGC). The percentage of flavonoids from the two branch pathways determines the sensory attributes of the wine to a certain extent. Except for the genetic factors, the accumulation of flavonoids in grapes is influenced by a number of external factors, such as crop level, viticulture practices, soil and other environmental factors, of which the climate impact is relatively obvious (Mateus et al., 2001; Downey et al., 2006). In terms of a particular cultivar, the accumulation of phenolic compounds depends largely on the terroir, such as vineyard soil, topography and climate (Gladstones, 2011). Vineyard altitude could result in variations in temperature, solar radiation and ultraviolet radiation, and these climate factors have been demonstrated to have a strong effect on the synthesis of phenolic compounds (Mateus et al., 2001; 2002; Berli et al., 2011). Studies have also examined the effects of vineyard altitude on phenolic and aromatic compounds in grapes and the wines produced (Mateus et al., 2001; 2002; Berli et al., 2013), but so far these studies have been concerned mainly with oceanic climate conditions such as the North European (like Portugal) and South American (such as Brazil and Argentina) climates. The data obtained do not necessarily reflect the accumulation pattern of flavonoids in grapes growing under a continental monsoon climate. This aspect is also of great importance in such a low-latitude and high-attitude region as the Yunnan plateau of south-western China, where there is a particular dry, cool valley microclimate under the typical continental monsoon climate.

The research on the formation of grape quality in high-altitude areas is not only fundamental to vineyard management strategies to acquire high-quality berries, but also helpful to enrich the theoretical understanding of the regulation of flavonoid synthesis. However, the reports on this topic are rather limited to date. In the present paper we aimed to investigate the accumulation characteristics of flavonoids in Vitis vinifera L. cv. Cabernet Sauvignon grape berries cultivated in different altitudes of the highland of Yunnan Province, China.

\section{MATERIALS AND METHODS \\ Sampling locations}

Sampling locations were three commercial vineyards of Vitis vinifera Cabernet Sauvignon of selected clones planted without rootstocks, at different altitudes in the county of Deqin, Yunnan Province of China. They are located at approximately 2900,2300 and $2150 \mathrm{~m}$ asl in Shuori $\left(28^{\circ} 34^{\prime} \mathrm{N}\right.$ and $\left.98^{\circ} 47^{\prime} \mathrm{E}\right)$, Meilishi $\left(27^{\circ} 49^{\prime} \mathrm{N}\right.$ and $\left.99^{\circ} 42^{\prime} \mathrm{E}\right)$ and Benzilan $\left(28^{\circ} 14^{\prime} \mathrm{N}\right.$ and $\left.99^{\circ} 18^{\prime} \mathrm{E}\right)$. The grapevines were all 10 years old and were trained on a vertical shoot-positioning (VSP) system arranged in north-south oriented rows spaced $2.0 \mathrm{~m}$ apart, with a distance of about $1.0 \mathrm{~m}$ between plants in each row. The three vineyards had similar types of soil and were subjected to similar viticulture management, including irrigation, fertiliser application, disease control and pruning. Each grapevine contained a main vine with 10 to 12 fruiting branches.

We also collected data on the main climate parameters in Deqin County, Yunnan Province in the grape-growing seasons of 2009 and 2010. The cumulative rainfall in the grape-growing season of 2009 was less than that in 2010 (223.9 mm vs. $253.6 \mathrm{~mm}$ ), and the average sunshine time in 2009 was longer in comparison with that in 2010 (298.3 $\mathrm{h}$ vs. $263.1 \mathrm{~h}$ ). The two vintages presented similar average temperatures $\left(13.5^{\circ} \mathrm{C}\right.$ vs. $\left.13.0^{\circ} \mathrm{C}\right)$ and average temperature differences between daytime and night time $\left(9.5^{\circ} \mathrm{C}\right.$ vs. $\left.8.9^{\circ} \mathrm{C}\right)$

\section{Grape materials}

The experimental samples were collected at the indicated stages from the Shuori and Benzilan vineyards in the grape berry-growing season of 2009, and the Shuori and Meilishi vineyards in 2010. The sampling time was fixed from 10:00 to 11:00 in the morning. To get a representative grape sample, we collected approximately 300 berries per vineyard from at least 100 clusters in different grapevines of different rows at random in each period, and excluded the ones on the edge. In the field, grape berries were kept on ice to prevent dehydration. In the laboratory, some of the grapes were used for the analysis of $\mathrm{pH}$ and total soluble solids (expressed in ${ }^{\circ}$ Brix). The changes in soluble solids, the $\mathrm{pH}$ of the must and sampling dates are shown in Table 1. The total sugar content of the berries was estimated by refractometric measurement of the total soluble solid contents of expressed juice from a representative sample of berries. The $\mathrm{pH}$ of the berries was measured in a $50 \mathrm{~g}$ sample that had been homogenised in a blender for 10 seconds. The remaining grapes were peeled. 
TABLE 1

Sampling period, $\mathrm{pH}$ and Brix of Cabernet Sauvignon berries from three vineyards.

\begin{tabular}{|c|c|c|c|c|c|c|c|c|}
\hline \multirow[b]{3}{*}{ WAFB } & \multicolumn{4}{|c|}{2009} & \multicolumn{4}{|c|}{2010} \\
\hline & \multicolumn{2}{|c|}{ Shuori } & \multicolumn{2}{|c|}{ Benzilan } & \multicolumn{2}{|c|}{ Shuori } & \multicolumn{2}{|c|}{ Meilishi } \\
\hline & pH & Brix & pH & Brix & pH & Brix & pH & Brix \\
\hline 4 & - & - & - & - & - & - & 2.35 & 4.33 \\
\hline 6 & 2.31 & 3.8 & 2.54 & 3.5 & 2.58 & 4.48 & - & - \\
\hline 8 & - & - & 2.49 & 6.2 & - & - & 2.54 & 4.43 \\
\hline 9 & 2.29 & 8.3 & - & - & - & - & - & - \\
\hline 10 & - & - & 2.85 & 13.7 & 2.66 & 9.46 & 2.78 & 10.18 \\
\hline 11 & 2.55 & 12.2 & - & - & - & - & & - \\
\hline 12 & 2.72 & 17.8 & 2.95 & 16.2 & 2.95 & 16.58 & 2.94 & 13.64 \\
\hline 13 & 3.05 & 20.1 & - & - & - & - & - & - \\
\hline 14 & - & - & 3.07 & 18.3 & - & - & - & - \\
\hline 16 & 3.15 & 22.5 & 3.31 & 22.7 & 3.19 & 22.1 & 3.30 & 25.81 \\
\hline
\end{tabular}

WAFB represents weeks after full bloom; “-” represents no sampling.

The skins were ground to a fine powder in liquid nitrogen, then lyophilised for $24 \mathrm{~h}$ at $-50^{\circ} \mathrm{C}$ using an LGJ-10 freezedryer and stored at $-40^{\circ} \mathrm{C}$ until extraction. Sampling was done similarly in consecutive years.

\section{Reagents}

Ethyl acetate, methanol, formic acid and sodium acetate (analytical grade) were produced by Beijing Chemical Reagent Plant (Beijing, China). All the standards and phloroglucinol were purchased from Sigma Chemical Co. (St. Louis, MO, USA). Methanol, formic acid, acetonitrile and acetic acid (HPLC grade) were products of the Fisher Co. (Fair Lawn, NJ, USA). Deionised water (18 M $\Omega$ resistivity) was prepared using a Millipore Milli-Q water purification system (Waters-Millipore Corporation, Milford, MA, USA). All other chemicals were purchased from Sigma-Aldrich Co. unless otherwise noted.

\section{Extraction of flavonoid compounds from grape skins}

Three groups of flavonoids were isolated from the grape skins according to Liang et al. (2013). For the extraction of anthocyanins, grape skin powder $(0.5 \mathrm{~g})$ was suspended in $10 \mathrm{~mL}$ of methanol containing $2 \%(\mathrm{v} / \mathrm{v})$ formic acid. The mixture was subjected to ultrasound treatment for $10 \mathrm{~min}$, followed by shaking in the dark at $180 \mathrm{rpm}$ for $30 \mathrm{~min}$ at $25^{\circ} \mathrm{C}$. The homogenate was centrifuged at $8000 \mathrm{rpm}$ and the supernatant was collected. The residue was extracted four times with the same solvent. The organic fraction was pooled and evaporated by a vacuum rotary evaporator at $30^{\circ} \mathrm{C}$. The concentrate was finally re-dissolved in $10 \mathrm{~mL}$ of $10 \%$ ethanol solution ( $\mathrm{pH} 3.7$ ).

For the isolation of flavonols, powdered grape skin $(2.00 \mathrm{~g})$ was macerated with $5 \mathrm{~mL}$ of distilled water and then mixed with $45 \mathrm{~mL}$ of ethyl acetate in darkness for $30 \mathrm{~min}$ at $25^{\circ} \mathrm{C}$. After the supernatant had been collected by centrifugation, the precipitate was re-extracted. This procedure was repeated four times. The pooled supernatant was evaporated by a rotary evaporator at $30^{\circ} \mathrm{C}$ until dryness. Finally, the residue was re-dissolved using $2 \mathrm{~mL}$ methanol.
With regard to the isolation of flavan-3-ols, we directly adopted acid degradation at $70^{\circ} \mathrm{C}$ to cleave proanthocyanidins into flavan-3-ol units in the presence of excess phloroglucinol (Liang et al., 2012a). In this situation, the data acquired should represent the total content of various flavan-3-ol units in the grape skins, including free monomers and the component units of their oligomers and polymers. The main extraction procedure was as follows: we mixed $0.005 \mathrm{~g}$ of ascorbic acid with $0.1 \mathrm{~g}$ of the dried skin powder, the objective of which was to protect flavan-3-ol units against oxidation in the process of extraction; the mixture was then added to $1 \mathrm{~mL}$ of phloroglucinol buffer $(0.5 \mathrm{~g}$ of phloroglucinol, $10 \mathrm{~mL}$ of methanol, $258 \mu \mathrm{L}$ of concentrated $\mathrm{HCl}$ ), and incubated in a shaking water bath at $70^{\circ} \mathrm{C}$ for 20 min; the extract subsequently was neutralised with $1 \mathrm{~mL}$ of sodium acetate $(200 \mathrm{mM}, \mathrm{pH} 7.5)$. The homogenate was centrifuged at $10000 \times g$ for $15 \mathrm{~min}$ and the supernatant was collected. The residue was extracted three times with the same phloroglucinol buffer. All the supernatants were pooled and used immediately for analysis.

All of the samples were filtered through $0.22 \mu \mathrm{m}$ filters prior to HPLC analysis.

\section{Analysis of individual flavonoid compounds}

Two millilitres of the extract per experimental unit was filtered through a $0.22 \mu \mathrm{m}$ pore size nylon membrane. The filtrate of $100 \mu \mathrm{L}$ was then injected into the HPLC-DAD system. An Agilent 1200 HPLC equipped with a G1322A degasser, a G1367C HiP-ALS, a G1312B bin pump, a G1316B TCC and a G1314C VWD were employed. The mass-spectrometric acquisition parameters were set as follows: drying gas flow, $10 \mathrm{~mL} / \mathrm{min}$; drying gas temperature, $325^{\circ} \mathrm{C}$; nebuliser pressure, $30 \mathrm{psi}$. The flavonols and flavan3 -ols were measured in negative ion mode, and anthocyanins were determined in positive ion mode. Details of the chromatographic conditions were as described previously (Li et al., 2011). 


\section{Qualitative and quantitative analyses of flavonoid compounds}

The identification of some compounds was carried out by comparing their mass spectrometry information, elution order and retention time with those of commercially available standards, which include quercetin, quercetin3-O-glucoside, quercetin-3-O-galactoside, kaempferol3-O-glucoside, myricetin, (+)-catechin, (-)-epicatechin, (-)-epigallocatechin, (-)-epicatechin-3-O-gallate, cyanidin3-O-glucoside, peonidin-3-O-glucoside, delphinidin3-O-glucoside, and malvidin-3-O-glucoside. Other flavonoid compounds without available standards were identified mainly by comparing molecular ions, product ions, and the elution orders of these compounds with those available in the published literature (Castillo-Muñoz et al., 2009; Liang et al., 2012b).

All flavonoid compounds were quantified according to the peak area through the external standard method. The relative concentrations of each anthocyanin and flavonol were quantified using malvidin-3-O-glucoside and quercetin as a standard respectively. The quantification of flavan-3ols was based on their corresponding standard components: $(+)$-catechin, (-)-epicatechin, (-)-epigallocatechin and (-)-epicatechin-3-O-gallate.

\section{Statistical analyses}

All the qualitative and quantitative analyses of flavonoids were performed in duplicate. Each data point, expressed as milligram equivalent of the respective standard per gram of dried grape skin, was the average of the data including two extraction replicates and two technological replicates per extraction. Two-way analysis of variance (ANOVA) was implemented to compare the effect of vineyard altitude and vintage on the accumulation of various components.

\section{RESULTS}

Flavonoid composition of skins during berry development A total of 40 flavonoid compounds were identified from the samples (Table 2), including 13 flavonols, four flavan-3-ols and 23 anthocyanins. The composition of flavonoids varied slightly with vintage and vineyard altitude. For example, syringetin-3-O-glucoside and delphinidin-3-O-(6-Ocoumaryl)-glucoside were detected only in 2009 and not in 2010; peonidin-3-O-(6-O-caffeoyl)-glucoside was detected only in the grapes from the higher altitude Shuori vineyard and not in Benzilan and Meilishi; some compounds, such as dihydroquercetin- $O$-hexoside, dihydroquercetin-(6- $O$ caffeoyl)-3-O-glucoside, peonidin-3,5- $O$-diglucoside and malvidin-3,5-O-diglucoside, were qualified only in some samples or at a certain stage, probably because most of these compounds presented the concentrations close to the detection limit. Interestingly, the diglucosides of anthocyanidins and pelargonidin-3-O-glucoside, which generally are thought to be absent in Vitis vinifera Cabernet Sauvignon grapes, were also found in the present grape samples.

Delphinidin-3,5-O-diglucoside could be quantified in most of the samples at a low concentration, while peonidin3,5-O-diglucoside was identified only in the Shuori grapes and malvidin-3,5-O-diglucoside only in the Meilishi grapes in the 2010 vintage. Pelargonidin-3-O-glucoside was also present in the Shuori and Benzilan grapes, probably as a result of the adaptability of this grape to extreme environmental conditions.

The total concentration of flavonoids roughly presented a declining trend along with berry development (Fig. 1). Compared to the Benzilan or Meilishi samples, the Shuori samples from the highest altitude generally contained a higher level of flavonoids from after véraison until full ripening. At commercial harvest, the total flavonoid concentration in the Shuori samples was significantly higher than that in Benzilan or Meilishi grapes. On the other hand, vintage greatly affected the production of flavonoids. Total concentration of flavonoids in Shuori grapes in 2010 was at least one-fold higher than that in 2009.

With regard to the three groups of flavonoids, the accumulation pattern of flavonols appeared to vary easily in terms of both altitude and vintage. But the grape berries from the higher-altitude Shuori vineyard had higher levels of flavonols when compared with the berries from Benzilan or Meilishi. At commercial harvest, the total flavonol concentration in the Shuori grapes cultivated at the highest altitude was 2.2 times higher than the Benzilan samples in 2009 (1.8 vs. $0.8 \mathrm{mg} / \mathrm{g})$, and 3.3 times higher than the Meilishi samples in 2010 (1.0 vs. $0.3 \mathrm{mg} / \mathrm{g}$ ) (Fig. 1), suggesting that high-altitude cultivation could promote the synthesis of flavonols.

As the main constituents of grape flavonoids, the variation in flavan-3-ols during berry development determines that of the total flavonoids to a greater extent. In the same vintage there were minor differences in the concentration of flavan-3ols between the grapes from different altitudes, whereas there was a great difference between the 2009 and 2010 growing seasons. At harvest point, the total flavan-3-ol concentration in the skins of grapes from Shuori was significantly higher than that observed in the Meilishi samples in 2010 (60.3 vs. $37.5 \mathrm{mg} / \mathrm{g}$ ), but no statistically significant differences were observed in 2009 (20.9 vs. 19.2 mg/g) (Fig. 1).

As for anthocyanins, the altitude of the vineyard had a significant effect on the accumulation of anthocyanin. The grape berries from the higher-altitude Shuori vineyard contained a higher level of anthocyanins. At commercial harvest, the total anthocyanin concentration in Shuori samples in particular was 1.2 times higher than that in the Benzilan samples in 2009 (25.6 vs. $20.0 \mathrm{mg} / \mathrm{g}$ ), and 2.3 times higher than Meilishi samples in 2010 (47.6 vs. $20.40 \mathrm{mg} / \mathrm{g}$ ) (Fig. 1).

\section{Flavonoids from different branch pathways}

To evaluate the influence of vineyard altitude on the $\mathrm{F}^{\prime} \mathrm{H}$ and $\mathrm{F}^{\prime}, 5^{\prime} \mathrm{H}$ branches of the flavonoid biosynthetic pathway, we calculated the concentrations of $3^{\prime}, 4^{\prime}$-substituted and $3^{\prime}, 4^{\prime}, 5^{\prime}$-substituted compounds respectively, in terms of each group of flavonoids (Fig. 2). In the grape berries growing in higher-altitude Shuori, the concentrations of flavonols and anthocyanins produced through either of the two branches were all significantly higher than the corresponding compounds in the Benzilan and Meilishi grapes in either 2009 or 2010. Separately, the concentrations of $3^{\prime}, 4^{\prime}$-substituted $(\mathrm{C}+\mathrm{EC}+\mathrm{ECG})$ or $3^{\prime}, 4^{\prime}, 5^{\prime}$-substituted (EGC) flavan-3-ols were similar between the vineyards in 2009 , but showed a 
great difference in 2010. At harvest point, the concentrations of various flavan-3-ols in the grapes skins from Shuori were significantly higher than those from Meilishi, suggesting that the variation in magnitude of flavan-3-ols by grape cultivation altitude was dependent on the growing season. In general, high-altitude cultivation favours the synthesis of flavonoids in the grape berry.

Regarding the percentage of $3^{\prime}, 4^{\prime}, 5^{\prime}$-substituted and $3^{\prime}, 4^{\prime}$-substituted flavonoids versus total flavonoids in the grape skins from different altitudes, it can be seen that the synthesis of the corresponding flavonoids through the $\mathrm{F}^{\prime}{ }^{\prime}{ }^{\prime} \mathrm{H}$ branch pathway was more than that from the $\mathrm{F}^{\prime} \mathrm{H}$

TABLE 2

Flavonoid compounds identified in the skins of Vitis vinifera Cabernet Sauvignon during the sampling period.

\begin{tabular}{|c|c|c|c|c|c|c|}
\hline \multirow{2}{*}{$\begin{array}{l}\text { Retention } \\
\text { time (min) }\end{array}$} & \multirow[t]{2}{*}{ Flavonoid compounds } & \multirow{2}{*}{$\begin{array}{l}{[\mathrm{M}]+(\text { fragment- }} \\
\text { MS2) }(\mathrm{m} / \mathrm{z})\end{array}$} & \multicolumn{2}{|c|}{2009} & \multicolumn{2}{|c|}{2010} \\
\hline & & & Shuori & Benzilan & Shuori & Meilishi \\
\hline & Flavonols & & & & & \\
\hline 6.99 & Dihydrokaempferol-3-O-glucoside & 449 (287) & + & + & + & + \\
\hline 7.55 & Kaempferol-3-O-galactoside & $447(285)$ & + & + & + & + \\
\hline 9.83 & Dihydroquercetin- $O$-hexoside & $465(339,301)$ & - & - & + & - \\
\hline 14.17 & Myricetin-3-O-galactoside & $479(317)$ & + & + & + & + \\
\hline 15.25 & Dihydroquercetin-(6-caffeoyl)-3-O-glucoside & $627(465,339,301)$ & + & - & - & - \\
\hline 18.78 & Dihydroquercetin-3'-O-rhamnoside & $449(285,303,151)$ & + & + & + & + \\
\hline 20.05 & Quercetin-3-O-glucuronide & $477(301)$ & + & + & + & + \\
\hline 21.56 & Quercetin-3-O-rutinoside & $609(301)$ & + & + & + & + \\
\hline 23.61 & Laricitrin-3-O-glucoside & $493(331)$ & + & + & + & + \\
\hline 29.43 & Isorhamnetin-3-O-galactoside & $477(315)$ & + & + & + & + \\
\hline 31.553 & Isorhamnetin-3-O-glucuronide & $477(315)$ & + & + & + & + \\
\hline 33.677 & Syringetin-3-O-glucoside & $507(345)$ & + & + & - & - \\
\hline & Flavan-3-ols & & & & & \\
\hline 14.24 & (-)-Epigallocatechin & $305(179,137)$ & + & + & + & + \\
\hline 18.50 & $(+)$-Catechin & $289(245)$ & + & + & + & + \\
\hline 32.91 & (-)-Epicatechin & $289(245)$ & + & + & + & + \\
\hline 46.90 & (-)-Epicatechin-3-O-gallate & $441(289,169)$ & + & + & + & + \\
\hline & Anthocyanin & & & & & \\
\hline 2.65 & Peonidin-3,5-O-diglucoside & $625(463,301)$ & - & - & + & - \\
\hline 2.84 & Delphinidin-3,5-O-diglucoside & $627(465,303)$ & + & + & + & + \\
\hline 3.84 & Delphinidin-3-O-glucoside & $465(303)$ & + & + & + & + \\
\hline 5.04 & Malvidin-3,5-O-diglucoside & $655(493,331)$ & - & - & - & + \\
\hline 5.47 & Cyanidin-3-O-glucoside & $449(287)$ & + & + & + & + \\
\hline 6.39 & Petunidin-3-O-glucoside & 479 (317) & + & + & + & + \\
\hline 7.52 & Pelargonidin-3-O-glucoside & $433(271)$ & + & + & + & - \\
\hline 8.86 & Peonidin-3-O-glucoside & $463(301)$ & + & + & + & + \\
\hline 9.70 & Malvidin-3-O-glucoside & $493(331)$ & + & + & + & + \\
\hline 11.02 & Delphinidin-3-O-acetylglucoside & $507(303,465)$ & + & + & + & + \\
\hline 14.40 & Cyanidin-3-O-acetylglucoside & $491(287,449)$ & + & + & + & + \\
\hline 15.73 & Petunidin-3-O-acetylglucoside & $521(317,479)$ & + & + & + & + \\
\hline 19.14 & Delphinidin-3-O-coumaroylglucoside & $611(303,465)$ & + & + & - & - \\
\hline 19.45 & Peonidin-3- $O$-acetylglucoside & $505(301,463)$ & + & + & + & + \\
\hline 20.29 & Malvidin-3-O-acetylglucoside & $535(331,493)$ & + & + & + & + \\
\hline 22.15 & Peonidin-3-O-caffeoylglucoside & $625(463,301)$ & + & - & + & - \\
\hline 22.64 & Cyanidin-3-O-coumaroylglucoside & $595(449,287)$ & + & + & + & + \\
\hline 22.96 & Malvidin-3-O-caffeoylglucoside & $655(493,331)$ & + & - & + & + \\
\hline 23.91 & Petunidin-3-O-coumaroylglucoside & $625(317,479)$ & + & + & + & + \\
\hline 25.53 & Peonidin-3-O-coumaroylglucoside & $609(463,301)$ & + & + & + & + \\
\hline 26.13 & Malvidin-3-O-coumaroylglucoside & $639(493,331)$ & + & + & + & + \\
\hline
\end{tabular}

Notes: "+" represents that this compound can be detected; "-" means it was not detected 
branch pathway (Fig. 3 A-D). In the same year, the percentage of either $3^{\prime}, 4^{\prime}, 5^{\prime}$-substituted or $3^{\prime}, 4^{\prime}$-substituted flavonoids was similar for the two vineyards, which revealed that highaltitude cultivation would result in a similar influence in the two branch pathways. Compared to the grapes harvested in these two years, the percentage of F3' $\mathrm{H}$-branch products in the 2010 grapes was bigger than that in 2009 (approximately $32.7 \%$ vs. $26.1 \%$ ), which means that vintage would alter the allocation of the assimilated carbon in the two branches of the flavonoid pathway. Considering the fact that the flavonoid concentration in the Shuori grapes of 2010 was much higher than that in 2009, we found that, in the 2010 Shuori grapes, the $\mathrm{F}^{\prime} \mathrm{H}$ branch synthesis was more up-regulated than that of the $\mathrm{F}^{\prime} 5^{\prime} \mathrm{H}$ branch.

To further illustrate the effects of vineyard altitude on the accumulation of flavonoids in the same branch pathway, we calculated the percentage of $\mathrm{F}^{\prime} \mathrm{H}$ (or F3' ${ }^{\prime} \mathrm{H}$ )-produced flavan-3-ols, flavonols and anthocyanins respectively, versus total F3'H (or F3'5'H) flavonoids (Fig. 3 E-F; the related actual concentrations are listed in Supplemental Table). At harvest point, the grape berries from the higher altitude Shuori vineyard achieved a larger proportion of cyanidin-type anthocyanins from the $\mathrm{F}^{\prime}{ }^{\prime} \mathrm{H}$ branch in the two years when compared to the Benzilan and Meilishi samples. Meanwhile, although quercetin-type and myricetin-type flavonols accounted for minor percentages, higher altitude conditions
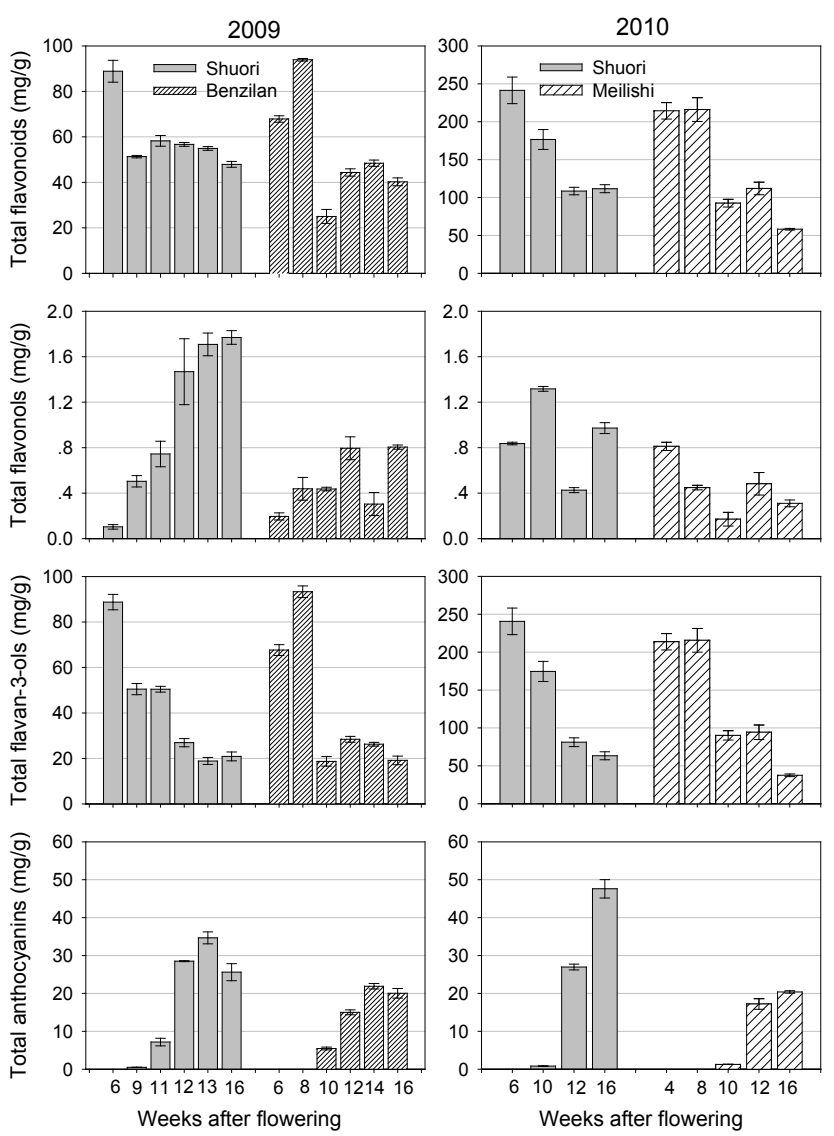

FIGURE 1

Accumulation of three groups of flavonoids in the skins of Cabernet Sauvignon grapes from different high altitudes in 2009 (left) and 2010 (right). increased the proportions of flavonols from both branches in the grape berry. In contrast, higher altitude cultivation resulted in a reduction in the proportions of flavan-3-ols from both of the branch pathways. These observations indicate that high-altitude cultivation largely enhances the synthesis of anthocyanins and flavonols in the two branch pathways, while, in terms of anthocyanins, the F3'H-produced cyanidin derivatives are improved more markedly by vineyard altitude than the delphinidin derivatives from the $\mathrm{F}^{\prime} 5^{\prime} \mathrm{H}$ branch. Compared with those from the other two regions, the grape berries from higher altitude Shuori contained much higher concentrations of cyanidin-3-O-glucoside, peonidin3-O-glucoside, cyanidin-3-O-(6-O-coumaryl)-glucoside,
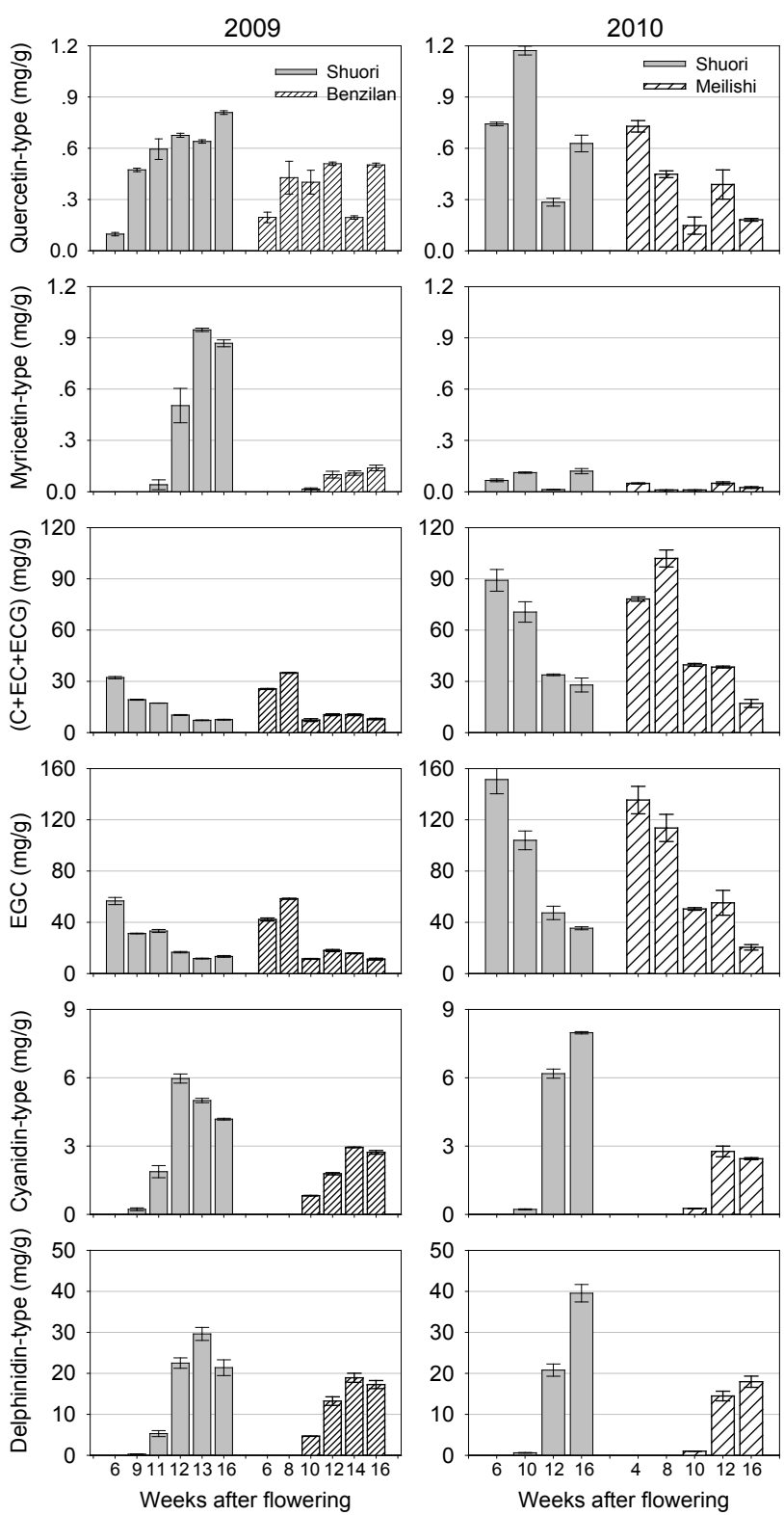

FIGURE 2

Accumulation of flavonols, flavan-3-ols and anthocyanins from two branches of flavonoid pathway in the skins of Cabernet Sauvignon grapes cultivated in different highaltitude vineyards in 2009 (two columns on the left) and 2010 (two columns on the right). 
cyanidin-3-O-(6-O-acetyl)-glucoside, malvidin-3-O-(6-Ocaffeoyl)-glucoside, petunidin-3-O- glucoside and myricetin-3-O-galactoside, etc.

\section{Composition and concentration of anthocyanins}

The anthocyanins identified in this experiment were classified as acylated and non-acylated forms according to the presence or absence of acyl moieties on the glucosides. As shown in Fig. 4, the grapes from the Shuori region had higher levels of acylated and non-acylated anthocyanins when compared with those from the other two vineyards respectively. As for the Shuori grapes, the accumulation of the two classes of anthocyanins both showed a first increase, followed by a decline in 2009 and a gradual increase in 2010 during berry

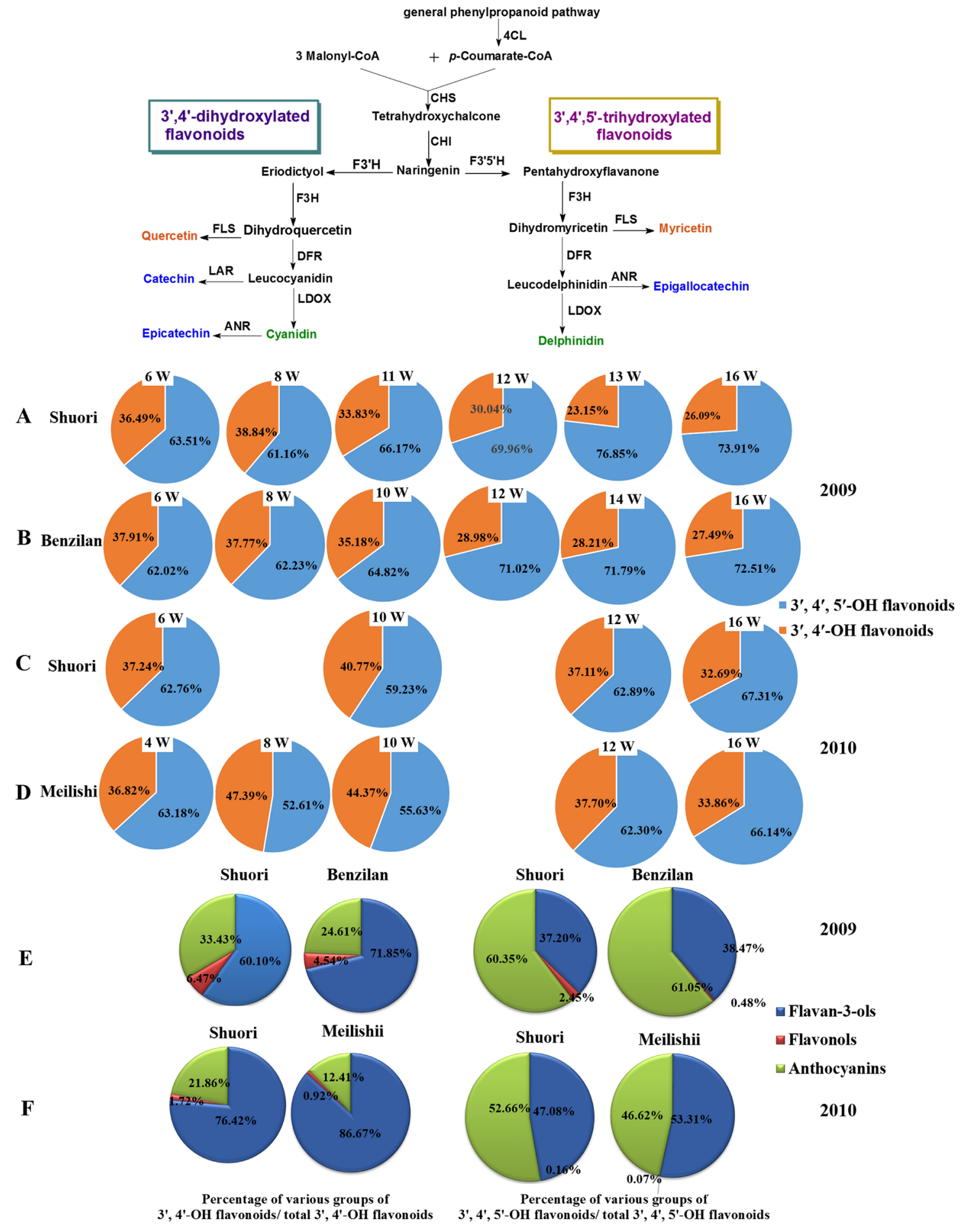

FIGURE 3

Concentration percentages of $3^{\prime}, 4^{\prime}, 5^{\prime}-\mathrm{OH}$ derivatives and $3^{\prime}, 4^{\prime}-\mathrm{OH}$ derivatives respectively, to total flavonoids (A, B, C and D), as well as concentration percentages of three groups of $3^{\prime}, 4^{\prime}-\mathrm{OH}$ derivatives respectively, to total $3^{\prime}, 4^{\prime}$-OH flavonoids in the F3' $\mathrm{H}$ branch and three groups of $3^{\prime}, 4^{\prime}, 5^{\prime}-\mathrm{OH}$ derivatives to total $3^{\prime}, 4^{\prime}, 5^{\prime}-\mathrm{OH}$ flavonoids in the F3'5' $\mathrm{H}$ branch (E and F) in the skins of Cabernet Sauvignon grapes from different high-altitude vineyards at commercial harvest in 2009 and 2010. 

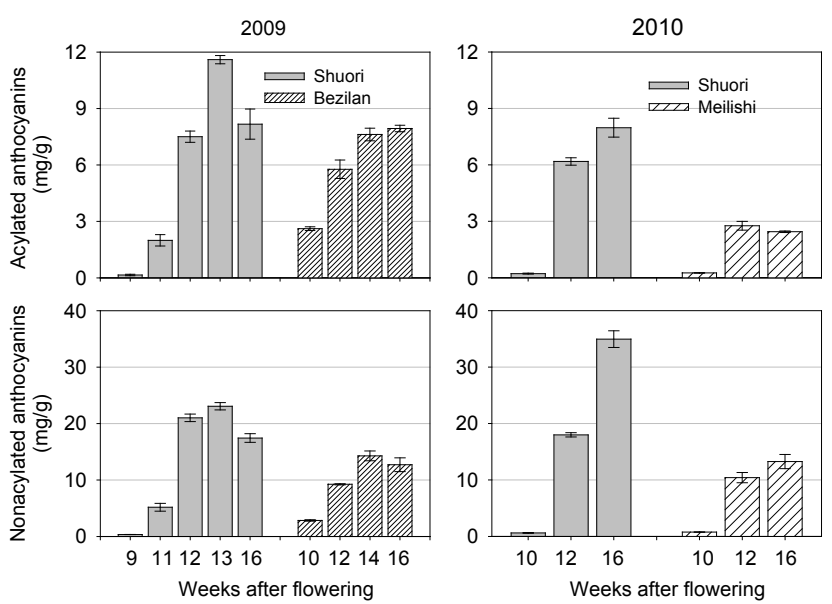

FIGURE 4

Accumulation of acylated and non-acylated anthocyanins in the skins of Cabernet Sauvignon grapes from different highaltitude vineyards in 2009 (left) and 2010 (right).

maturation. Meanwhile, in the Benzilan and Meilishi grapes, those two classes of compounds both displayed a rapid rise and then a slight decline in 2009 and 2010. In terms of proportion, acylated anthocyanins in grapes from Shuori occupied a lower proportion in comparison with those from Benzilan and Meilishi, which indicates that higher altitude cultivation would promote a more pronounced increase in non-acylated anthocyanins than acylated ones.

\section{PCA and ANOVA}

Principal component analysis (PCA) was used to compare the extent of the influence of vineyard altitude and vintage. Fourteen evaluation parameters were composed of the concentrations of cyanidin-type, delphinidin-type, acetyled, coumaryled, caffeoyled, non-acylated and total anthocyanins; quercetin-type, myricetin-type, kaempferoltype and total flavonols; $\mathrm{C}+\mathrm{EC}+\mathrm{ECG}$; EGC and total flavan3 -ols. The data for these parameters came from the samples of Shuori and Benzilan in the 2009 vintage as well as the samples of Shuori and Meilishi in 2010, with two replicates, taking into account the sampling dates from 12 to 16 weeks after flowering. PCA explained $80.44 \%$ of the total variance in the first two principal components, with $51.69 \%$ for PC1 and $28.75 \%$ for PC2 respectively (Fig. 5). The scatter plot of the first two principal components illustrated the differences between grapevine cultivation altitudes and between vintages (Fig. 5 A).The corresponding loading plot demonstrated the relative weight of the variables (Fig. 5 B). As shown in Fig. 5, PC1 could clearly distinguish the grapes growing in relatively higher altitude Shuori from ones in Benzilan and Meilishi, while PC2 could obviously differentiate the grapes harvested in the 2009 vintage from ones in 2010. The Shuori grapes of the 2009 vintage were all scattered in the fourth quadrant, with a positive score in $\mathrm{PC} 1$, corresponding to the highest concentrations of various classes of flavonols, coumarylated and caffeoylated anthocyanins at maturity. The Shuori grapes growing in 2010 were characterised by the highest concentrations of various classes of anthocyanins (apart from coumarylated and caffeoylated anthocyanins), as these samples were all concentrated in the first quadrant. The Meilishi grapes had the higher level of flavan-3-ols in comparison to the grapes collected from Shuori and Benzilan in 2009, which was reflected by the distribution of these sample in the second quadrant, with a negative score in PC1 and a positive score in PC2. On the whole, a very high level of flavonols and anthocyanins is representative of the grapes growing in the Shuori location at $2900 \mathrm{~m}$ (asl). However, based on PCA, we found it difficult to determine which influence - vintage or altitude - was the biggest.

To better evaluate the impact of altitude and vintage on the accumulation of individual flavonoids and the interaction between both factors, we performed two-way ANOVA (Table 3). Among the 36 compounds detected in this study, 32 exhibited significantly differential levels depending on the vineyard altitude of the grapevines, and 29 depending on vintage. The accumulation of 27 individual flavonoids was significantly affected by both factors. Of these compounds, peonidin-3-O-(6-O-acetyl)-glucoside was not affected by either cultivation altitude or vintage. It is roughly concluded from the $F$-value that most of the flavonols and anthocyanins were influenced more by cultivation altitude than vintage, and the opposite situation was observed for flavan-3-ols, which is in good agreement with the above results. From the two-way ANOVA we also found that various anthocyanins in the grape berries, except for peonidin-3-O-(6-O-acetyl)glucoside, all were significantly different in the concentration owing to the cultivation altitude of the grapevines.

\section{DISCUSSION}

The present study shows that vineyard altitude plays a positive role in the accumulation of flavonoids, especially that of anthocyanins and flavonols, in Cabernet Sauvignon grape skins, which is similar to the results of previous studies (Mateus et al., 2001; 2002). This could be related to the high-altitude climate. A few observations have mentioned that high-altitude cultivation strongly alters solar radiation, temperature and other environmental factors around grapevines (Mateus et al., 2002). A higher altitude generally corresponds to stronger sunlight (Cortell \& Kennedy, 2006; Fanzone et al., 2010), lower temperature and humidity (Ojeda et al., 2002; Castellarin et al., 2007), greater temperature difference between day and night, and more extreme environmental conditions (Mateus et al., 2001), all of which are important factors affecting the production of flavonoids. Flavonoids, especially anthocyanins and flavonols, are also considered to be effective absorbers of UV-B (Zidorn et al., 2005; Cortell \& Kennedy, 2006). The abundant UV-B radiation in high-altitude regions could result in the accumulation of anthocyanins and flavonols in grape skins (Cortell \& Kennedy, 2006; Fanzone et al., 2010), which have been demonstrated by the current study. Our previous study also indicated that UV-responsive production of anthocyanins is partly a consequence of the increase in carbon supply (Zhang et al., 2012). In the present study, in the same growing season, vineyard altitude showed limited influence on the accumulation of flavan-3-ols in grape berries. Mateus et al. (2001) observed that lower altitude is an important factor favouring the biosynthesis of higher concentrations 

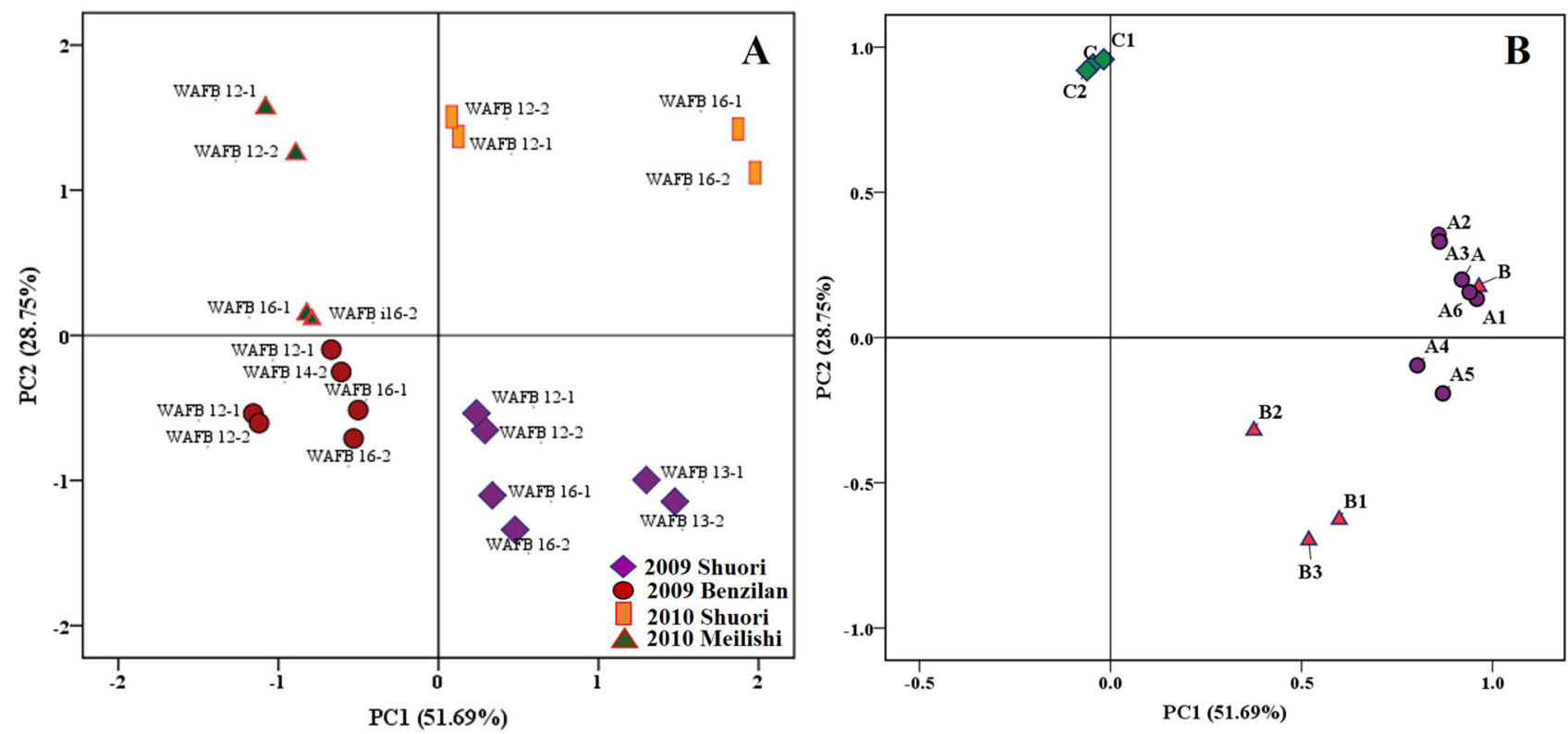

FIGURE 5

Principal component analysis based on various flavonoids in the skins of Cabernet Sauvignon grapes from 12 weeks after full bloom to commercial harvest: (A) score scatter plot; (B) loading plot. WAFB stands for weeks after full bloom, and the first number after it means the sampling time and the second indicates the replicate of the samples.

of grape-skin flavan-3-ol monomers, procyanidin dimers, trimer $\mathrm{C} 1$, as well as total extractable proanthocyanidins. The inconsistent conclusions are probably related to the great difference in grapevine cultivation altitudes between the two studies. In our experiment, the grape berries grew at an altitude of 2150 to $2900 \mathrm{~m}$ above sea level, and in the former report the grapes came from an altitude of 100 to $300 \mathrm{~m}$ (Mateus et al., 2001). Our former studies also showed that no elevation, even statistically significant suppression, was observed when the 11-week-old grapes were exposed to UV irradiation (Zhang et al., 2013). Furthermore, the biosynthesis of flavan-3-ols in grapes occurs mainly before véraison. There seems to be a limited impact of vineyard altitude on the synthesis of flavan-3-ols.

In terms of the $\mathrm{F}^{\prime} \mathrm{H}$-mediated and $\mathrm{F}^{\prime} 5^{\prime} \mathrm{H}$-mediated branches of the flavonoid synthetic pathway, the present study indicates the an increase in cultivation altitude would greatly stimulate the accumulation of anthocyanins and flavonols from both branches simultaneously. From the view of the same branch, cyanidin-type anthocyanins together with quercetin-type and myricetin-type flavonols are dramatically more enhanced by high-altitude cultivation than other types of compounds in a high-altitude region like Shuori. Flavonols are a group of important co-pigment molecules that could stabilise colour to some extent during winemaking (Schwarz et al., 2005). It can be concluded that high altitude conditions promote the quality of the grape berry as related to colour and co-pigmentation in wine.

Wine colour is an important sensory attribute and depends largely on the extractability and composition of anthocyanins from grape skins during winemaking (Ryan \& Revilla, 2003). The colour attributes of anthocyanins vary with the substituents of the B-ring. The maximum absorbance wavelengths of cyanidin-type (two hydroxyl groups on the B-ring; also called 3'4'-substituted) and delphinidin-type (three hydroxyl groups on the B-ring) anthocyanins are approximately at 516 and $525 \mathrm{~nm}$ respectively, and they tend to give $\mathrm{red} / \mathrm{magenta}$ and violet/blue colorations. Based on the present data, the grapes growing in higher altitude Shuori, in relation to Benzilan and Meilishi, theoretically should endow red wine with a deeper colour, especially more red/magenta, as they contain a higher concentration of anthocyanins and a higher proportion of cyanidin-type forms. Additionally, the acylation in the glycosyl moiety of anthocyanins could improve the stability or/and blue shift. It has been proposed that aromatic acylation makes anthocyanins more stable and bluer by intramolecular stacking of the anthocyanins with polyphenols, and that the acetylation of anthocyanin does not change the colour, but enhances the pigment solubility in water by protecting glycosides from enzymatic degradation and stabilising anthocyanin structures (Honda \& Saito, 2002; He et al., 2012; Flamini et al., 2013). In this study, although the grapes growing in the Shuori vineyard contained much higher levels of both acylated and non-acylated anthocyanidins when compared to the grapes from Benzilan and Meilishi, this influence consists more in the non-acylated form than the acylated form. Accordingly, the high-altitude cultivation of grapevines appears to offer relatively limited promotion of the stability of wine colour.

It is noteworthy that both the diglucosides of anthocyanidins and pelargonidin-3-O-glucoside were detected at a very low level in the grapes from the lowlatitude and high-altitude regions of south-western China. It has commonly been accepted that diglucosides of anthocyanidin scarcely exist in Vitis vinifera grapes like Cabernet Sauvignon (Picariello et al., 2014). In our previous analyses of grape berries from the wine-producing region of western China, the existence of diglucosides of anthocyanidin repeatedly was demonstrated by mass spectrometry evidence (unpublished data). Additionally, we also have confirmed in 
TABLE 3

$F$ ratios and $p$ values in two-way analysis of variance regarding effects of both altitude and vintage on accumulation of individual flavonoids.

\begin{tabular}{|c|c|c|c|c|c|c|}
\hline \multirow[b]{2}{*}{ Flavonoid compounds } & \multicolumn{2}{|l|}{ Altitude } & \multicolumn{2}{|l|}{ Vintage } & \multicolumn{2}{|c|}{ Interaction } \\
\hline & $F$ & $p$ & $F$ & $p$ & $F$ & $p$ \\
\hline \multicolumn{7}{|l|}{ Flavonols } \\
\hline Quercetin- $O$-hexoside & 491.799 & 0.000 & 326.846 & 0.000 & 2.356 & 0.200 \\
\hline Laricitrin-3-O-glucoside & 8.569 & 0.043 & 8.569 & 0.043 & 7217.687 & 0.000 \\
\hline Kaempferol- $O$-hexoside & 147.868 & 0.000 & 0.212 & 0.669 & 18.829 & 0.012 \\
\hline Myricetin-3-O-galactoside & 4260.290 & 0.000 & 3498.811 & 0.000 & 2225.484 & 0.000 \\
\hline Dihydroquercetin-3-O- (6-caffeoyl)- glucoside & 431.542 & 0.000 & 431.542 & 0.000 & 431.542 & 0.000 \\
\hline Dihydroquercetin-3-O-rhamnoside & 188.578 & 0.000 & 13.431 & 0.021 & 6.267 & 0.067 \\
\hline Quercetin-3-O-glucuronide & 73.264 & 0.001 & 0.202 & 0.676 & 0.015 & 0.909 \\
\hline Quercetin-3-O-rutinoside & 28.296 & 0.006 & 0.218 & 0.665 & 0.006 & 0.943 \\
\hline Laricitrin-3-O-glucoside & 0.312 & 0.606 & 70.147 & 0.001 & 21.593 & 0.010 \\
\hline Isorhamnetin-3-O-galactoside & 33.788 & 0.004 & 1366.807 & 0.000 & 20.674 & 0.010 \\
\hline Syringetin-3-O-glucoside & 0.001 & 0.975 & 859.122 & 0.000 & 0.001 & 0.975 \\
\hline \multicolumn{7}{|l|}{ Flavan-3-ols } \\
\hline (-)-Epigallocatechin & 165.544 & 0.000 & 566.515 & 0.000 & 91.199 & 0.001 \\
\hline (-)-Epicatechin & 14.089 & 0.020 & 83.821 & 0.001 & 14.809 & 0.018 \\
\hline (-)-Epicatechin-3-O-gallate & 21.009 & 0.010 & 88.162 & 0.001 & 30.011 & 0.005 \\
\hline$(+)$-Catechin & 6.209 & 0.067 & 292.384 & 0.000 & 4.838 & 0.093 \\
\hline \multicolumn{7}{|l|}{ Anthocyanins } \\
\hline Peonidin-3, 5-O-glucoside & 315.191 & 0.000 & 315.191 & 0.000 & 315.191 & 0.000 \\
\hline Malvidin-3, 5-O-diglucoside & 155.255 & 0.000 & 155.255 & 0.000 & 155.255 & 0.000 \\
\hline Dephinidin-3-O-glucoside & 147.840 & 0.000 & 15.201 & 0.001 & 65.930 & 0.001 \\
\hline Petunidin-3-O-glucoside & 363.498 & 0.000 & 217.904 & 0.000 & 185.833 & 0.000 \\
\hline Malvidin-3-O-glucoside & 2102.040 & 0.000 & 964.895 & 0.000 & 1010.973 & 0.000 \\
\hline Dephinidin-3-O-(6-O-acetyl)-glucoside & 132.024 & 0.000 & 94.244 & 0.001 & 80.274 & 0.000 \\
\hline Petunidin-3-O-(6-O-acetyl)-glucoside & 155.153 & 0.000 & 95.085 & 0.001 & 94.098 & 0.001 \\
\hline Dephinidin-3-O-(6-O-coumaryl)-glucoside & 8.076 & 0.047 & 993.197 & 0.000 & 8.076 & 0.047 \\
\hline Malvidin-3-O-(6-O-acetyl)-glucoside & 17.048 & 0.015 & 18.328 & 0.013 & 27.543 & 0.006 \\
\hline Malvidin-3-O-(6-O-caffeoyl)-glucoside & 41.256 & 0.003 & 6.777 & 0.060 & 18.978 & 0.012 \\
\hline Petunidin-3-O-(6-O-coumaryl)-glucoside & 434.287 & 0.000 & 242.916 & 0.000 & 1096.387 & 0.000 \\
\hline Malvidin-3-O-(cis-6-O-coumaryl)-glucoside & 39.550 & 0.003 & 2.433 & 0.194 & 10.056 & 0.034 \\
\hline Malvidin-3-O-(trans-6-O-coumaryl)-glucoside & 40.996 & 0.003 & 13.532 & 0.021 & 36.599 & 0.004 \\
\hline Cyanidin-3-O-glucoside & 427.459 & 0.000 & 137.079 & 0.000 & 150.843 & 0.000 \\
\hline Peonidin-3-O-glucoside & 998.324 & 0.000 & 237.347 & 0.000 & 324.050 & 0.000 \\
\hline Cyanidin-3-O-(6-O-acetyl)-glucoside & 183.344 & 0.000 & 19.799 & 0.011 & 77.437 & 0.001 \\
\hline Peonidin-3-O-(6-O-acetyl)-glucoside & 3.695 & 0.127 & 2.233 & 0.209 & 3.507 & 0.134 \\
\hline Peonidin-3-O-(6-O-caffeoyl)-glucoside & 324.528 & 0.000 & 5.352 & 0.082 & 5.352 & 0.082 \\
\hline Cyanidin-3-O-(6-O-coumaryl)-glucoside & 58.292 & 0.002 & 18.180 & 0.013 & 29.887 & 0.005 \\
\hline Peonidin-3-O-(cis-6-O-coumaryl)-glucoside & 74.743 & 0.001 & 105.671 & 0.001 & 0.001 & 0.983 \\
\hline Peonidin-3-O-(trans-6- $O$-coumaryl)-glucoside & 32.908 & 0.005 & 1.842 & 0.246 & 34.844 & 0.004 \\
\hline Pelargonidin-3-O-glucoside & 99.153 & 0.001 & 6246.274 & 0.000 & 99.153 & 0.001 \\
\hline
\end{tabular}

Notes: The shaded data show a $p$ value of more than 0.05 , which represents no statistically significant difference at the 0.05 level. The shaded compounds mean that the effects of cultivation altitude on these compounds is larger than that of vintage. 


\section{SUPPLEMENTARY TABLE}

Contents of total $3^{\prime}, 4^{\prime}-\mathrm{OH}$ flavonoids and $3^{\prime}, 4^{\prime}, 5^{\prime}-\mathrm{OH}$ flavonoids, as well as three groups of $3^{\prime}, 4^{\prime}-\mathrm{OH}$ and $3^{\prime}, 4^{\prime}, 5^{\prime}-\mathrm{OH}$ derivatives in the skins of Cabernet Sauvignon grapes from different high-altitude vineyards at commercial harvest in 2009 and 2010.

\begin{tabular}{lllll}
\hline \multirow{2}{*}{ Flavonoids } & \multicolumn{1}{c}{$\mathbf{2 0 0 9}$ Shuori } & \multicolumn{1}{c}{ Benzilan } & Shuori & Meilishi \\
\cline { 2 - 5 } 3',4'-OH flavonoids & $12.53 \pm 0.250$ & $11.05 \pm 0.433$ & $36.48 \pm 1.108$ & $19.71 \pm 0.425$ \\
3',4',5'-OH flavonoids & $35.41 \pm 0.750$ & $29.17 \pm 0.505$ & $75.13 \pm 1.176$ & $38.51 \pm 1.397$ \\
Cyanidin-type & $4.18 \pm 0.004$ & $2.72 \pm 0.008$ & $7.98 \pm 0.005$ & $2.45 \pm 0.040$ \\
Delphinidin-type & $21.37 \pm 0.019$ & $17.26 \pm 0.020$ & $39.53 \pm 0.003$ & $17.95 \pm 0.369$ \\
Quercetin-type & $0.81 \pm 0.010$ & $0.50 \pm 0.011$ & $0.63 \pm 0.049$ & $0.18 \pm 0.008$ \\
Myricetin-type & $0.87 \pm 0.006$ & $0.14 \pm 0.016$ & $0.12 \pm 0.015$ & $0.03 \pm 0.004$ \\
C+EC+ECG & $7.54 \pm 0.235$ & $7.93 \pm 0.414$ & $27.88 \pm 1.054$ & $17.09 \pm 0.377$ \\
EGC & $13.33 \pm 0.724$ & $11.22 \pm 0.469$ & $35.37 \pm 1.158$ & $20.45 \pm 1.124$
\end{tabular}

Notes: Results are expressed in $\mathrm{mg} / \mathrm{g}$; values represent means of duplicate determination.

a prior study the presence of pelargonidin-3-O-glucoside in the berry skins of Cabernet Sauvignon and Pinot Noir (Vitis vinifera L.) (He et al., 2010), which is in agreement with the current observation. It is proposed that the diversity of the composition of anthocyanins in Cabernet Sauvignon grapes could result from the extreme ecological and climatic conditions of highlands like Deqin. However, more research is needed to explain this phenomenon.

\section{CONCLUSIONS}

On the whole, some uncommon anthocyanin components, such as three diglucosides of anthocyanidins and pelargonidin-3-O-glucoside, were detected in the 'Cabernet Sauvignon' grape berries in the present study. At this high altitude, both anthocyanin and flavonol profiling are altered by the higher cultivation altitude of grapevines, even in the same vintage. A higher altitude tends to promote the production of anthocyanins and flavonols, particularly cyanidin-type or non-acylated anthocyanins. In contrast, the concentration of flavan-3-ols was altered less by altitude. With regard to flavan-3-ols, the impact of vintage was greater than cultivation altitude. This study shows that grapes from this high altitude have good winemaking potential.

\section{LITERATURE CITED}

Berli, F.J., Alonso, R., Bressan-Smith, R. \& Bottini, R., 2013. UV-B impairs growth and gas exchange in grapevines grown in high altitude. Physiol. Plant. 149, 127-140.

Berli, F.J., Fanzone, M., Piccoli, P. \& Bottini, R., 2011. Solar UV-B and $\mathrm{ABA}$ are involved in phenol metabolism of Vitis vinifera $\mathrm{L}$. increasing biosynthesis of berry skin polyphenols. J. Agric. Food. Chem. 59, 48744884 .

Castellarin, S., Matthews, M., Gaspero, G. \& Gambetta, G., 2007. Water deficits accelerate ripening and induce changes in gene expression regulating flavonoid biosynthesis in grape berries. Planta 227, 101-112.

Castillo-Muñoz, N., Fernández-González, M.N., Gómez-Alonso, S., García-Romero, E. \& Hermosín-Gutiérrez, I., 2009. Red-color related phenolic composition of Garnacha Tintorera (Vitis vinifera L.) grapes and red wines. J. Agric. Food. Chem. 57, 7883-7891.

Castillo-Muñoz, N., Gómez-Alonso, S., García-Romero, E. \& HermosínGutiérrez, I., 2007. Flavonol profiles of Vitis vinifera red grapes and their single-cultivar wines. J. Agric. Food. Chem. 55, 992-1002.
Cortell, J.M. \& Kennedy, J.A., 2006. Effect of shading on accumulation of flavonoid compounds in (Vitis vinifera L.) Pinot Noir fruit and extraction in a model system. J. Agric. Food. Chem. 54, 8510-8520.

Davies, K.M. \& Schwinn, K.E., 2003. Transcriptional regulation of secondary metabolism. Funct. Plant Biol. 30, 913-925.

Dixon, R.A., Xie, D.-Y. \& Sharma, S.B., 2005. Proanthocyanidins - a final frontier in flavonoid research? New Phytol. 165, 9-28.

Downey, M.O., Dokoozlian, N.K. \& Krstic, M.P., 2006. Cultural practice and environmental impacts on the flavonoid composition of grapes and wine: A review of recent research. Am. J. Enol. Vitic. 57, 257-268.

Fanzone, M.N., Peña-Neira, A.L., Jofré, V., Assof, M. \& Zamora, F., 2010. Phenolic characterization of Malbec wines from Mendoza Province (Argentina). J. Agric. Food Chem. 58, 2388-2397.

Flamini, R., Mattivi, F., Rosso, M.D., Arapitsas, P. \& Bavaresco, L., 2013. Advanced knowledge of three important classes of grape phenolics: Anthocyanins, stilbenes and flavonols. Int. J. Mol. Sci. 14, 19651-19669.

Gawel, R., 1998. Red wine astringency: A review. Aust. J. Grape Wine Res. 4, 74-95.

Gladstones, J.S., 2011. Wine, terroir and climate change. Wakefield Press, Kent Town, South Australia.

Gómez-Míguez, M., González-Manzano, S., Escribano-Bailón, M.T., Heredia, F.J. \& Santos-Buelga, C., 2006. Influence of different phenolic copigments on the color of malvidin 3-glucoside. J. Agric. Food Chem. 54, $5422-5429$

He, F., He, J.J., Pan, Q.H., \& Duan, C.Q., 2010. Mass-spectrometry evidence confirming the presence of pelargonidin-3-O-glucoside in the berry skins of Cabernet Sauvignon and Pinot Noir (Vitis vinifera L.). Aust. J. Grape Wine Res. 16, 464-468.

He, F., Liang, N.-N., Mu, L., Pan, Q.-H., Wang, J., Reeves, M.J. \& Duan, C.-Q., 2012. Anthocyanins and their variation in red wines I. Monomeric anthocyanins and their color expression. Molecules 17, 1571-1601.

Honda, T. \& Saito, N., 2002. Recent progress in the chemistry of polyacylated anthocyanins as flower color pigments. Heterocycles 56, 633-692.

Koyama, K., Ikeda, H., Poudel, P.R. \& Goto-Yamamoto, N., 2012. Light quality affects flavonoid biosynthesis in young berries of Cabernet Sauvignon grape. Phytochem. 78, 54-64.

Li, Z., Pan, Q., Jin, Z., Mu, L. \& Duan, C., 2011. Comparison on phenolic compounds in Vitis vinifera cv. Cabernet Sauvignon wines from five winegrowing regions in China. Food Chem. 125, 77-83. 
Liang, N.-N., He, F., Pan, Q.-H., Wang, J., Reeves, M.J. \& Duan, C.-Q., 2012a. Optimization of sample preparation and phloroglucinol analysis of Marselan grape skin proanthocyanidins using HPLC-DAD-ESI-MS/MS. S. Afr. J. Enol. Vitic. 33, 122-131.

Liang, N.-N., He, F., Bi, H.-Q., Duan, C.-Q., Reeves, M.J. \& Wang, J., 2012 b. Evolution of flavonols in berry skins of different grape cultivars during ripening and a comparison of two vintages. Eur. Food Res. Technol. 235, 1187-1197.

Liang, N.-N., Pan, Q.-H., He, F., Wang, J., Reeves, M.J. \& Duan, C.-Q., 2013. Phenolic profiles of Vitis davidii and Vitis quinquangularis species native to China. J. Agric. Food. Chem. 61, 6016-6027.

Mateus, N., Machado, J.M. \& De Freitas, V., 2002. Development changes of anthocyanins in Vitis vinifera grapes grown in the Douro Valley and concentration in respective wines. J. Sci. Food Agric. 82, 1689-1695.

Mateus, N., Marques, S., Gonçalves, A.C., Machado, J.M. \& De Freitas, V., 2001. Proanthocyanidin composition of red Vitis vinifera varieties from the Douro Valley during ripening: Influence of cultivation altitude. Am. J. Enol. Vitic. 52, 115-121.

Obreque-Slier, E., Pena-Neira, A., Lopez-Solis, R., Zamora-Marin, F., Ricardo-da Silva, J.M. \& Laureano, O., 2010. Comparative study of the phenolic composition of seeds and skins from Carmenere and Cabernet Sauvignon grape varieties (Vitis vinifera L.) during ripening. J. Agric. Food. Chem. 58, 3591-3599.

Ojeda, H., Andary, C., Kraeva, E., Carbonneau, A. \& Deloire, A., 2002. Influence of pre- and postveraison water deficit on synthesis and concentration of skin phenolic compounds during berry growth of Vitis vinifera cv. Shiraz. Am. J. Enol. Vitic. 53, 261-267.

Picariello, G., Ferranti, P., Chianese, L. \& Addeo, F., 2012. Differentiation of Vitis vinifera L. and hybrid red grapes by matrix-assisted laser desorption/ ionization mass spectrometry analysis of berry skin anthocyanins. J. Agric. Food. Chem. 60, 4559-4566.
Picariello, G., Ferranti, P., Garro, G., Manganiello, G., Chianese, L., Coppola, R. \& Addeo, F., 2014. Profiling of anthocyanins for the taxonomic assessment of ancient purebred $V$. vinifera red grape varieties. Food Chem. $146,15-22$.

Ryan, J.-M. \& Revilla, E., 2003. Anthocyanin composition of Cabernet Sauvignon and Tempranillo grapes at different stages of ripening. J. Agric. Food. Chem. 51, 3372-3378.

Santos-Buelga, C. \& Scalbert, A., 2000. Proanthocyanidins and tannin-like compounds - nature, occurrence, dietary intake and effects on nutrition and health. J. Sci. Food Agric. 80, 1094-1117.

Schwarz, M., Picazo-Bacete, J.J., Winterhalter, P. \& Hermosín-Gutiérrez, I., 2005. Effect of copigments and grape cultivar on the color of red wines fermented after the addition of copigments. J. Agric. Food. Chem. 53, 8372 8381 .

Winkel-Shirley, B., 2001. Flavonoid biosynthesis. A colorful model for genetics, biochemistry, cell biology, \& biotechnology. Plant Physiol. 126, 485-493.

Zarrouk, O., Francisco, R., Pinto-Marijuan, M., Brossa, R., Santos, R.R., Pinheiro, C., Costa, J.M., Lopes, C. \& Chaves, M.M., 2012. Impact of irrigation regime on berry development and flavonoids composition in Aragonez (Syn. Tempranillo) grapevine. Agric. Water Manage. 114, 18-29.

Zhang, Z.-Z., Che, X.-N., Pan, Q.-H., Li, X.-X., \& Duan, C.-Q., 2013. Transcriptional activation of flavan-3-ols biosynthesis in grape berries by UV irradiation depending on developmental stage. Plant Sci. 208, 64-74.

Zhang, Z.-Z., Li, X.-X., Chu, Y.-N., Zhang, M.-X., Wen, Y.-Q., Duan, C.Q. \& Pan, Q.-H., 2012. Three types of ultraviolet irradiation differentially promote expression of shikimate pathway genes and production of anthocyanins in grape berries. Plant Physiol. Biochem. 57, 74-83.

Zidorn, C., Schubert, B. \& Stuppner, H., 2005. Altitudinal differences in the contents of phenolics in flowering heads of three members of the tribe Lactuceae (Asteraceae) occurring as introduced species in New Zealand. Biochem. Syst. Ecol. 33, 855-872. 